\title{
Evaluation of reversible and irreversible degradation rates of polymer electrolyte membrane fuel cells tested in automotive conditions
}

\author{
Pawel Gazdzicki $^{1 *}$, Jens Mitzel ${ }^{1}$, Daniel Garcia Sanchez ${ }^{1}$, Mathias Schulze ${ }^{1}$, K. Andreas Friedrich $^{1,2}$ \\ ${ }^{1}$ German Aerospace Center, Institute of Engineering Thermodynamics, Pfaffenwaldring 38-40, 70569 Stuttgart, \\ Germany \\ ${ }^{2}$ University of Stuttgart, Institute of Energy Storage, Pfaffenwaldring 31, 70569 Stuttgart, Germany
}

*Corresponding author

email address: pawel.gazdzicki@dlr.de (P. Gazdzicki)

\section{Highlights:}

- Discrimination between reversible and irreversible degradation

- Determination of voltage loss rates at different loads in a single durability test

- Quantitative evaluation of reversible voltage decay by linear-exponential function

- Recovery of reversible voltage losses by shutdown recovery procedure

Keywords: Polymer electrolyte membrane fuel cell, automotive, durability, irreversible degradation, reversible degradation, performance recovery

\section{ABSTRACT}

This work provides single cell durability tests of membrane electrode assemblies in dynamic operation regularly interrupted by recovery procedures for the removal of reversible voltage losses. Degradation rates at different loads in one single test can be determined from these tests. Hence, it is possible to report degradation rates versus current density instead of a single degradation rate value. A clear discrimination between reversible and irreversible voltage loss rates is provided. The irreversible degradation rate can be described by a linear regression of voltage values after the recovery steps. Using voltage values before refresh is less adequate due to possible impacts of reversible effects. The reversible contribution to the voltage decay is dominated by an exponential decay after restart, eventually turning into a linear one. A linear-exponential function is proposed to fit the reversible voltage degradation. Due to this function, the degradation behavior of an automotive fuel cell can be described correctly during the first hours after restart. The fit parameters decay constant, exponential amplitude and linear slope are evaluated. Eventually, the reasons for the 
voltage recovery during shutdown are analyzed showing that ionomer effects in the catalyst layer and/or membrane seem to be the key factor in this process.

\section{INTRODUCTION}

One of the most serious issues of the modern society in the last decades is the greenhouse gas emission caused by the use of fossil energy sources. Hydrogen, a non-fossil and regenerative fuel, is expected to play an important role in future energy conversion. The corresponding electrochemical energy conversion technologies, i.e. fuel cells that convert hydrogen and oxygen to electricity with water being the only exhaust product, are considered as a promising solution to contribute to the reduction of emission of environmental pollutants in the near future. Recently, significant progress has been achieved toward meeting the challenging cost and performance targets required for the use of polymer electrolyte membrane fuel cells (PEMFCs) in automotive applications. However, one of the main issues hindering the widespread commercialization of PEMFCs is the limited durability of membrane electrode assemblies (MEAs) caused by numerous chemical and physical degradation phenomena [1-4]. Upon long-term operation of PEMFCs all MEA components, such as membrane, electrodes and gas diffusion layers (GDLs), may undergo physical and chemical changes leading to a reduction of the cell performance over time [5]. In the case of the (perfluorosulfonic acid) membranes and ionomers the chemical degradation due to peroxide radical formation is usually observed [6-8]. Corresponding effects are membrane thinning or crack/pinhole formation $[5,9,10]$ leading to increased hydrogen crossover and eventually to fuel cell failure. Furthermore, the ionic conductivity of the membrane can be decreased by side chain cutting of the polymer [11]. The resulting increase of the ohmic losses over time can significantly reduce the fuel cell performance and efficiency. Typical degradation phenomena observed in the electrodes are loss of electrochemically active surface area due to catalyst particle growth or dissolution [12] and corrosion of the carbon support $[13,14]$ leading to increased ohmic resistance and activation losses. The degradation of the GDL is often due to the loss of hydrophobic agents like polytetrafluorethylene (PTFE) resulting in increased water management issues and increased reactant mass transport resistance of the cell [15]. All these different degradation phenomena, well described in the review papers by de Bruijn et al. [5] as well as by Schmittinger and Vahidi [6], have negative influence on the power density of the cell.

To overcome these problems at the European level and to support the market introduction of fuel cells, numerous projects addressing degradation issues have been recently funded by the Fuel Cells and Hydrogen Joint Undertaking of the European Commission ${ }^{1}$. A question which arises in this context is how to reliably assess if specific project targets regarding the improvement of MEA durability have been reached. To answer this question common and quantitative procedures to describe fuel cell degradation (voltage loss over time) are needed. However, a uniform method to describe fuel cell degradation is currently not established.

To obtain knowledge of the degradation behavior of a certain MEA it is of great interest to know the voltage decay rates at different current densities. However, in the literature dealing with

\footnotetext{
${ }^{1}$ Among others the FCH JU projects IMPACT (Grant 303452), Stack-Test (Grant 303445) and SecondAct (Grant 621216) are worth to be mentioned here covering automotive and stationary applications as well as stack testing procedures.
} 
degradation, the voltage decay rates are mainly reported for a single specific load only $[10,14,16]$. Moreover, reporting a single value for the degradation rate is not unambiguous if no indication about consideration of reversible and irreversible losses is provided. In order to discriminate between reversible and irreversible degradation specific tests with regular interruptions of the cell operation by a refresh procedure are needed such as performed by Kongkanand et al. [8]. Such non-continuous operation is typical for automotive applications and is therefore in line with real operation conditions.

Numerous papers $[2,5,6,8,17]$ address degradation under different operation conditions as well as the underlying degradation mechanisms. The degradation rates are often determined by a linear regression of the voltage values of a long-term operated PEMFC ignoring the effects of interruptions by shutdown [17]. For a cell continuously operated for multiple thousands of hours this approach allows an approximate estimation of the degradation, but specific information about irreversible and reversible degradation is lost. Publications clearly discriminating between reversible and irreversible degradation in PEMFC have been published by Kongkanand et al. [8], Kundu et al. [10], Enz et al. [14], Zang et al. [16] and Schulze et al. [15]. In the case of direct methanol fuel cells studies particularly focusing on the discrimination between reversible and irreversible degradation have been published by Cha et al. [18] and Bresciani et al. [19]. Kundu et al. [10] reported that irreversible degradation can be estimated from the linear part of the linear-exponential decay (approx. $50 \mathrm{~h}$ after start of continuous operation) occurring in constant operation conditions, i.e. from voltage values before performing the recovery of reversible losses. As turned out in our study, this approach is only applicable if reversible degradation is constant over time. An alternative, which is discussed in subsection 3.2, is performing a linear regression of the voltage values after the refresh steps.

To the best of our knowledge, up to now no paper focuses on the specific determination of degradation rates in general and in particular on the determination of degradation rates from durability test performed under dynamical conditions. This paper, dealing with the determination of degradation rates for automotive applications, is a contribution to this topic providing and comparing possibilities to evaluate voltage loss rates in standardized durability tests.

\section{EXPERIMENTAL}

Single cell tests were performed using gold coated stainless steel cells $\left(5 \times 5 \mathrm{~cm}^{2}\right.$ active area, single serpentine flow field). The in-house developed test stands controlled by programmable logic controllers allow automatic control of the input and output conditions, such as the pressure, temperature, gas flow rates and humidity. A commercial electronic load from Zentro Elektrik was used. The cells were operated at $80^{\circ} \mathrm{C}$ cell temperature, $50 \%$ relative humidity (RH) and 1.5 bar (absolute) pressure at gas outlets. The cells were fed with $\mathrm{H}_{2}(5 \mathrm{~N}$ purity) and pressurized and filtered air. All durability experiments were conducted at constant $\mathrm{H}_{2}$ and air flows of 260 and $830 \mathrm{ml} \mathrm{min}^{-1}$, respectively, corresponding to stoichiometric ratios of 1.5 and 2.0 for a current density of $1 \mathrm{~A} \mathrm{~cm}^{-2}$. The relative humidity of the inlet gasses was set by adjusting the temperature of the bubblers used as gas humidifiers. To avoid water condensation in the humid gases, the gas connector tubes were held at temperatures $5^{\circ} \mathrm{C}$ above the cell temperature. Before starting the durability test and before first diagnostics the MEAs were conditioned for several hours at $1 \mathrm{~A} \mathrm{~cm}^{-2}$ followed by a shutdown overnight (approx. $12 \mathrm{~h}$ ). After subsequent start up and a further conditioning of $2 \mathrm{~h}$ electrochemical characterization was performed and the degradation test was started. Voltage data and test bench parameters were acquired every $10 \mathrm{~s}$. 
Two MEAs were used in this paper. MEA1 is a developmental low Pt-loaded $\left(0.25 \mathrm{mg}_{\mathrm{pt}} / \mathrm{cm}^{2}\right.$ total) perfluorosulfonic acid membrane based MEA from the European project IMPACT (Grant 303452). MEA2 is a commercial product from Johnson Matthey Fuel Cells. As GDL, in both cases, a modified Sigracet 25BC was used with improved hydrophobic treatment from the European project DECODE (Grant 213295). The performance curves of both MEAs recorded at begin of test (BoT) are provided in Figure 1. The performance curves were measured at 1.5 and 2.0 stoichiometry for $\mathrm{H}_{2}$ and air, respectively, with minimum flows of $52 \mathrm{ml} \mathrm{min}^{-1}$ and $166 \mathrm{ml} \mathrm{min}^{-1}$.

The durability of the MEAs was tested using the European harmonized fuel cell dynamic load cycle (FC-DLC) developed in the European Stack-Test project (Grant 303445) and available in Ref [20,21].

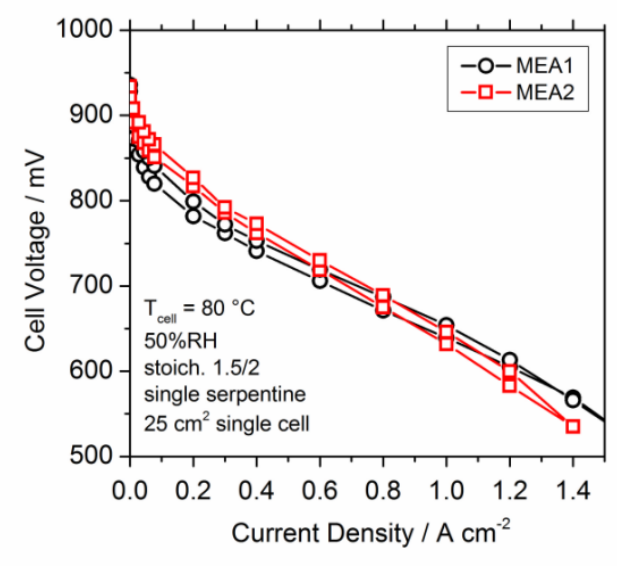

Figure 1: Performance curves of MEA1 and MEA2 at BoT.

\section{RESULTS AND DISCUSSION}

The Results and Discussion section is subdivided into four subsections. In subsection 3.1 the durability test of MEA1 is described. In subsection 3.2 the determination of irreversible degradation rates is discussed and degradation rates of MEA1 and for comparison also of MEA2 are provided. In subsection 3.3 the focus is on the evaluation of the reversible degradation rates based on the test performed with MEA1. Eventually, the shutdown procedure for the recovery of reversible voltage losses is analyzed based on tests performed using MEA2.

\subsection{Durability test}

To evaluate the voltage degradation process in automotive conditions, MEA1 was systematically tested using the FC-DLC protocol $[20,21]$. The current density profile of two subsequent cycles of this protocol (each lasting 20 minutes) is provided in Figure 2 (A) along with the recorded cell voltage. It is noted that this test performed at constant flow rates of $260\left(\mathrm{H}_{2}\right)$ and $830 \mathrm{ml} \mathrm{min}^{-1}$ (air) corresponding to respective stoichiometric ratios of 1.5 and 2.0 at the maximum current density of $1 \mathrm{~A} \mathrm{~cm}^{-2}$ represents an accelerated stress test (AST). In other words, excessive drying of the MEA due to water removal by the high gas flows occurs especially during the first 15 minutes of each cycle where the load values are low. Consequently, the presented durability test is an AST including both load cycling as well as humidity cycling which is particularly stressing for the membrane. 


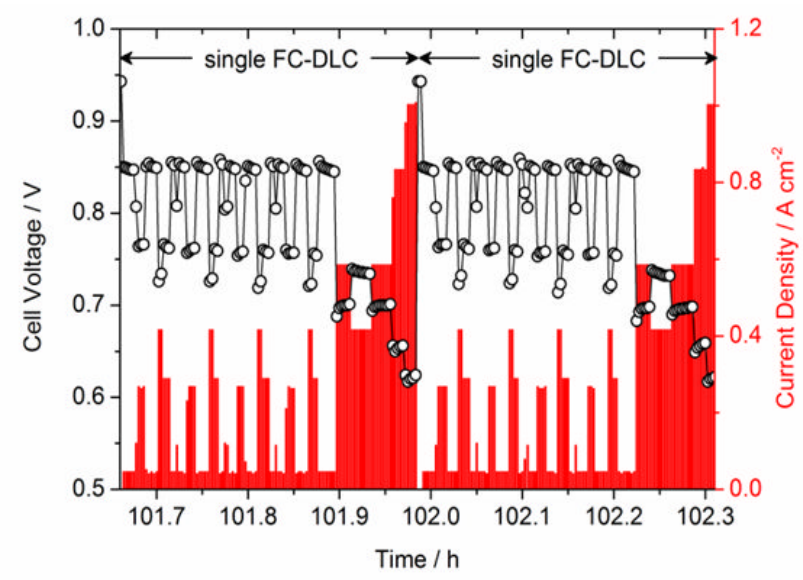

\section{(A)}

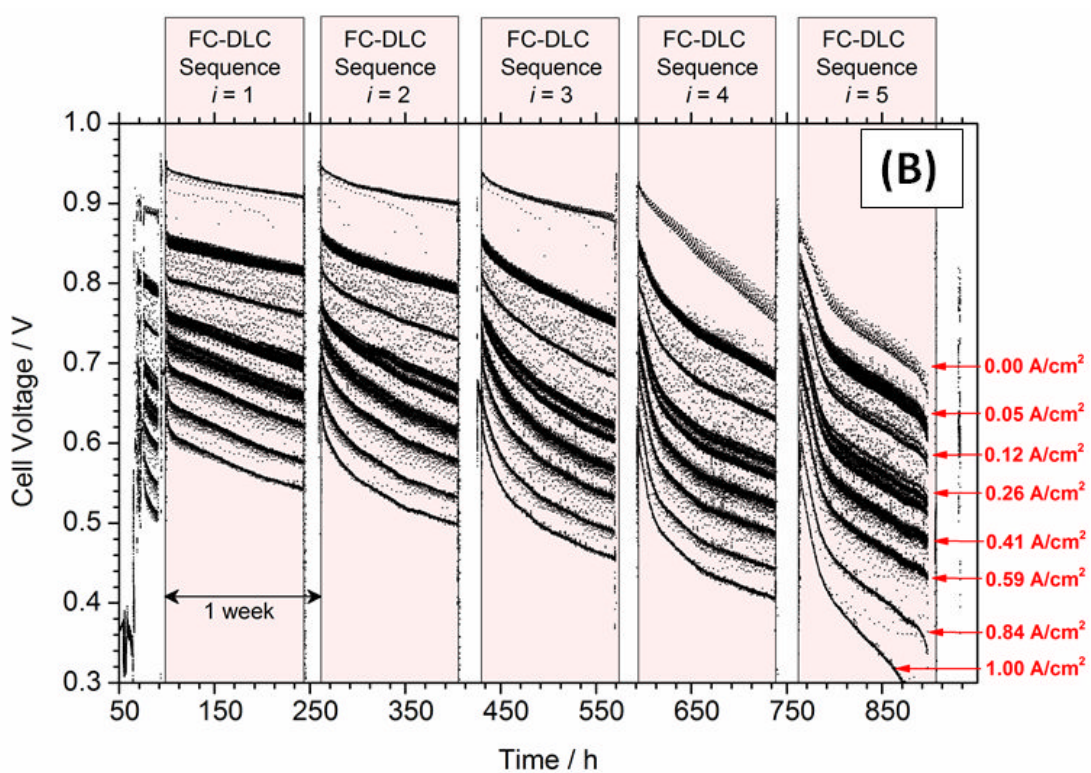

Figure 2: (A): The load profile of two subsequent FC-DLCs $[20,21]$ (as measured by the test bench) and the response of the corresponding single cell voltage of MEA1 $\left(25 \mathrm{~cm}^{2}\right)$. (B): A single FC-DLC durability test of MEA1. After each cycling sequence $i$ follow an electrochemical characterization and a shutdown for recovery of reversible losses. After subsequent startup of the cell and before starting the next sequence, the cell is again characterized. At the right side of the plot current density values belonging to the different voltage levels are indicated (compare panel A).

The entire durability test provided Figure 2 (B) consists of five periods of one week each. Each period covers a continuous FC-DLC sequence with 6 days duration followed by a one day period for cell characterization, performance recovery, and restart. The continuous FC-DLC operation within each of the five periods is called a "FC-DLC sequences" (indicated by the colored boxes in the figure) and is labelled by the index $i=1 \ldots 5$. After each sequence $i$ the following procedure was performed: (i) electrochemical characterization, (ii) shutdown for approximately $12 \mathrm{~h}$, (iii) start up, (iv) electrochemical characterization after all temperatures reached their target values. The shutdown recovery procedure is analyzed in subsection 3.4.

Note that the test in Figure 2 (B) corresponds to a single durability test of MEA1. Due to the test duration of hundreds of hour which is three orders of magnitude longer than an individual FC-DLC the test looks like a quasi-simultaneous acquisition of voltage values at different loads (indicated on the right side of the figure). Hence, the test allows the determination of quasi-performance curves every 20 minutes (duration of a single FC-DLC) without interruption of the cell operation that may interfere with the MEA leading to a partial recovery of reversible voltage losses. 
Apparently, the voltage decay becomes faster from one sequence to another which is in agreement with a previous study by Schulze et al. [15]. In sequence $i=4$ and especially in sequence $i=5$ the voltage loss rates at open circuit voltage $(\mathrm{OCV})\left(0.00 \mathrm{~A} \mathrm{~cm}^{-2}\right)$ are particularly strong due to membrane degradation and failure evidenced by an increase of $\mathrm{H}_{2}$ crossover current by a factor larger than ten at end of test (EOT). The increase of $\mathrm{H}_{2}$ crossover at EoT may be caused by severe membrane thinning or pinhole formation due to chemical degradation as well as by mechanical crack formation due to the humidity cycling during the FC-DLC sequences using constant reactant flows [22]. This membrane damage enables the formation of so-called mixed electrode potentials that are known to reduce the OCV [23].

Interestingly, at the beginning of the $5^{\text {th }}$ sequence the OCV becomes significantly increased compared to its value at the end of sequence $i=4$ meaning that the impact of the irreversible membrane failure depends on cell conditions and exhibits a dynamical behavior. It seems that drying during the FC-DLC sequence enhances the effect of the failure and humidification expected during the recovery leads to a reduction of its effect. Such sealing of pinholes by water has been reported by Kreitmeier et al. $[24,25]$.

The voltage losses during the individual sequences can be considered as an initially exponential-like decay followed by a constant decay as qualitatively described by Kundu et al.[10]. The voltage values after each shutdown are significantly higher than before the shutdown. Consequently, the voltage losses occurring during each sequence $i$ can be at least partially recovered. Hence, the overall voltage degradation can be separated into a fast and partially recoverable performance loss (reversible degradation) occurring under continuous operation and a slow permanent performance loss (irreversible degradation). The irreversible degradation rate corresponds to a linear regression of the voltage values just after the shutdown steps, i.e. the voltage values at the beginning of each sequence $i$. For a further description of reversible and irreversible degradation rates the reader is also referred to Ref. [18].

The performance curves of MEA1 recorded at BoT and after each recovery step are plotted in Figure 3 (A). The performance curves recorded before each recovery are provided in Figure 3 (B). In panel (C) the differential resistance $R_{\text {diff }}(j)=d U / d j$ is plotted. It represents the impedance of the examined single cell and is determined by the slope of the performance curve at the presented current densities [26]. When comparing the BoT performance curve and the curve after the first recovery, the cell performance shows a slight increase which is likely due to incomplete breaking-in of the MEA at BoT. The maximum cell performance, measured after the first refresh, equals $0.86 \mathrm{~W} \mathrm{~cm}^{-2}$ at $1.5 \mathrm{~A}$ $\mathrm{cm}^{-2}$. After this maximum, a gradual decrease of the performance measured after the refresh steps is observed until the $4^{\text {th }}$ refresh, i.e. before the start of sequence $i=5(755 \mathrm{~h})$. The sudden drop of performance between the $4^{\text {th }}$ refresh $(755 \mathrm{~h})$ and the $5^{\text {th }}$ refresh $(890 \mathrm{~h}$, EoT) is dramatic in the entire current density range in agreement with a failure of the MEA already described above. According to the performance curve after the $4^{\text {th }}$ refresh this failure is not dramatic until $755 \mathrm{~h}$, but it clearly starts developing already during the FC-DLC sequence $i=4$ as concluded from the strong voltage decay at OCV during this sequence observed in Figure 2 (B). These performance curves obtained after refresh are affected by irreversible degradation while the reversible performance loss is recovered by the refresh procedure.

The performance curves recorded before each refresh, shown in Figure 3 (B), are influenced by reversible and irreversible degradation. The changes from one curve to another are larger than in 
panel $(A)$ which is in line with the observation that reversible degradation is faster than the irreversible one. The slope of the performance curves for high current densities, however, is virtually identical in panels $(\mathrm{A})$ and $(\mathrm{B})$ as concluded from the almost constant differential resistances at $1.0 \mathrm{~A}$ $\mathrm{cm}^{-2}$ (see Figure $3(C)$ ) which means that the ohmic region of the performance curve is not affected by reversible degradation. In contrast, the voltage values at current densities lower $0.4 \mathrm{~A} \mathrm{~cm}^{-2}$, including OCV, are strongly affected by reversible degradation. This aspect is related to the significant increase of the differential resistance during the FC-DLC sequences, as shown in Figure 3 (C) for 0.2 and $0.4 \mathrm{~A} \mathrm{~cm}^{-2}$. The reversibility is demonstrated by the strong decrease of the differential resistance and the recovered performance in the performance curves after refresh.
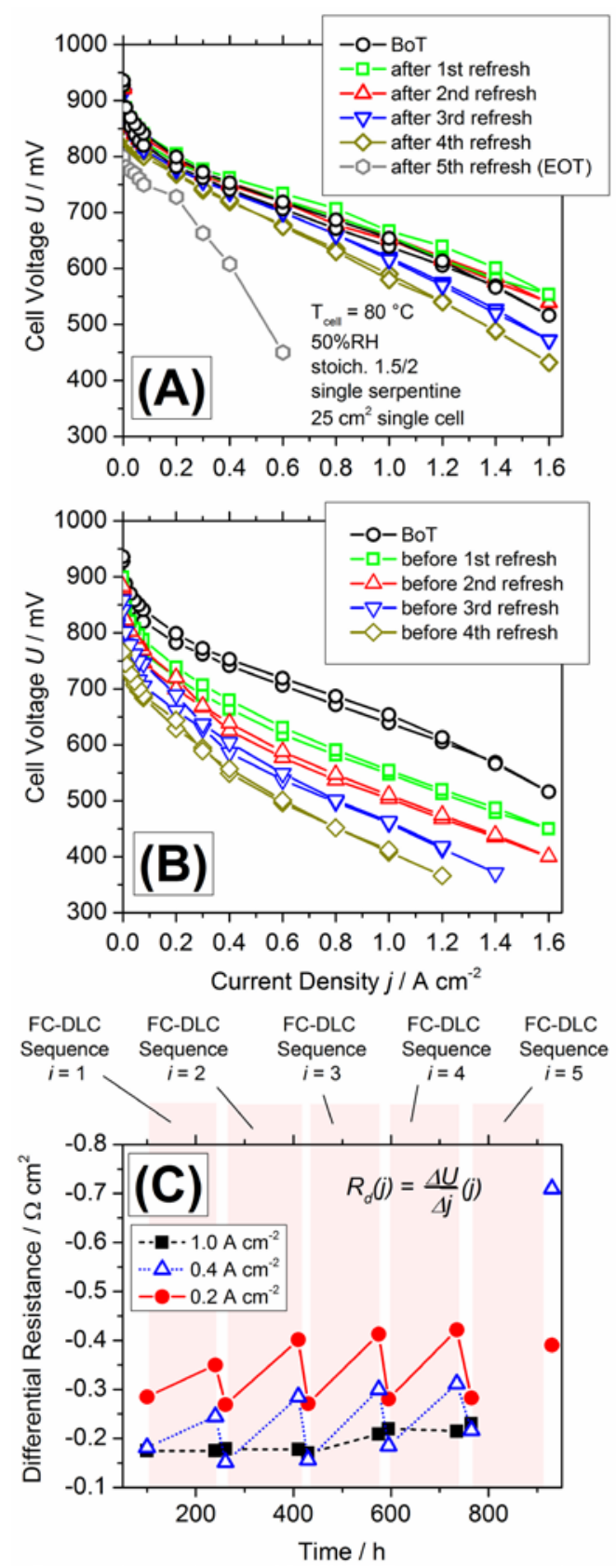

Figure 3: Performance curves of MEA1 acquired at BoT and after each refresh step (A) and before each refresh step (B). (C) shows the differential cell resistance (impedance) calculated from the performance curves for different current densities. After sequence $i=\mathbf{4}$ no performance curve was measured due to extraordinarily low cell voltages due to membrane failure. 


\subsection{Description of Irreversible Voltage Loss Rates}

As mentioned above, irreversible voltage loss rates can be evaluated using regular recovery of reversible voltage losses by the shutdown procedure. For the determination of the irreversible voltage loss rate at least two possibilities should be taken into account as indicated in Figure 4 (A). The first one is the calculation of the irreversible voltage loss rate using the voltage values directly after the refresh procedure; this approach should be generally applicable since reversible contributions are eliminated. An alternative is the calculation of the degradation rate based on voltage values just before the shutdown procedure. This possibility is only reliable if the reversible degradation is constant from one sequence to another(compare top and bottom panel of Figure 4 (A)). Otherwise a mixture of reversible and irreversible degradation is determined.

Both methods were applied to determine the degradation rates at different loads of the FC-DLC test in Figure 2 (B). To avoid the contribution of the drastic voltage drop in sequence $i=5$ due to a failure of the MEA, only data from sequences $i=1 \ldots 4$ were used for further analysis. The corresponding voltage loss rates plotted versus the current density (voltage loss diagram) are depicted in Figure 4 (B). Generally, the discrepancy between the degradation rates determined by the two methods is due to the fact that the rate obtained from voltage values before refresh contains a non-constant reversible contribution over time. This means that while the irreversible degradation (determined from voltages after refresh) is constant from one sequence to another, the reversible degradation which contributes to the voltage values before refresh increases from one sequence to another.

In Figure 4 (C) an analogous voltage loss diagram obtained from a $700 \mathrm{~h}$ durability test of MEA2 (see supporting information Figure S1) is provided for comparison. MEA2 was tested with the FC-DLC protocol with shutdown interruptions every $100-200 \mathrm{~h}$. Apparently, for MEA2 the two approaches to calculate the voltage loss rates (using voltage values before and after refresh) lead to similar results. In other words, in the case of MEA2 the reversible degradation is constant over time. Consequently, both degradation rates, calculated from voltage values before and after the refresh steps, correspond to the irreversible degradation. For MEA1, on the other hand, only the voltage values after the refresh can be used to determine irreversible degradation.
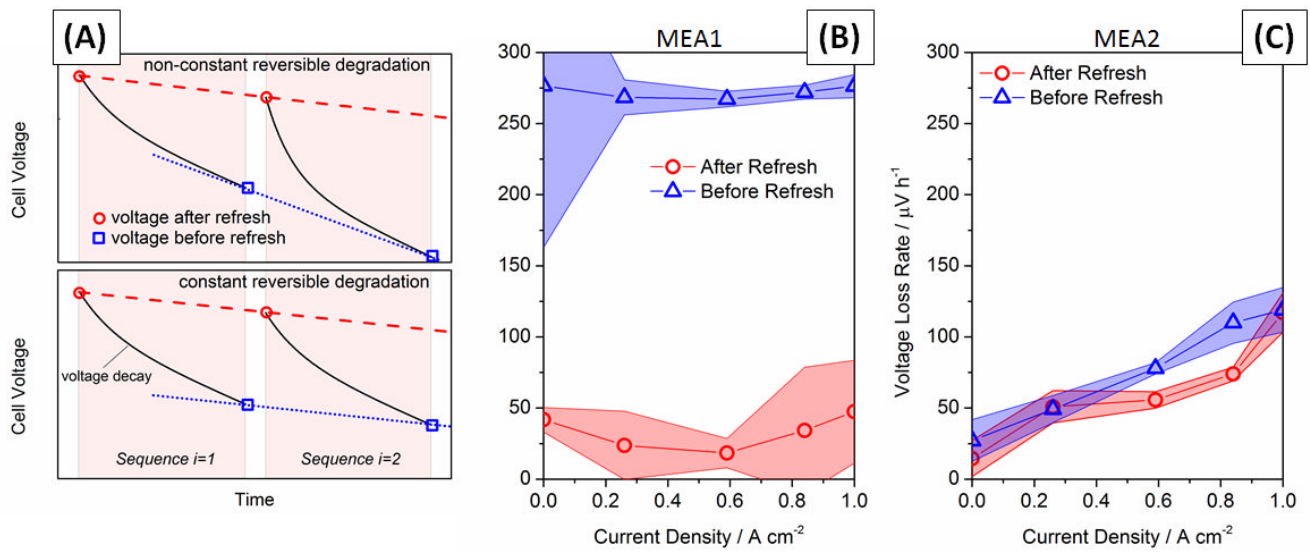

Figure 4: (A) Scheme showing two possibilities to determine irreversible degradation rates using voltage values before refresh (squares) and after refresh (circles). The top and the bottom panel show the case in which the reversible degradation is non-constant and constant, respectively. (B) Voltage loss rate curves of MEA1 using linear regressions of voltage values before and after refresh. $(C)$ Voltage loss rate curves of MEA2 determined from a FC-DLC durability test using linear regression of voltage values before and after refresh. The colored areas correspond to the errors of the linear regressions. 


\subsection{Description of Reversible Voltage Loss Rates}

The cell voltage recorded at $1 \mathrm{~A} \mathrm{~cm}^{-2}$ during the degradation test of MEA1 is shown in Figure $5(\mathrm{~A})$. For a moment, the other loads are ignored for better clarity of presentation. The individual FC-DLC sequences, labelled by colored boxes, show a non-linear voltage decay which is recoverable to a large extent. Evidently, a linear regression even using a superposition of two linear functions with different slopes is inappropriate to fit the data of the individual FC-DLC sequences. Hence, it is proposed to fit the voltage behavior using the linear-exponential function

$U_{i}(t)=a_{i}-b_{i}\left(t-t_{i}\right)+c_{i} \exp \left(-\lambda_{i}\left(t-t_{i}\right)\right)$

(Equation 1)

which corresponds to the red solid curves in Figure 5 (A). $t_{i}$ corresponds to the start times of the FCDLC sequences. The fit parameters describing the reversible degradation of the voltage is the slope $b_{i}$ of the linear part of $U_{i}(t)$, the decay constant $\lambda_{i}$ which is reciprocal to the time constant of the exponential decay, and the amplitude $c_{i}$ of the exponential decay (thereby, at the start of each sequence $\left.U_{i}\left(t=t_{i}\right)=a_{i}+c_{i}\right)$. For the sake of clarity the linear part (dashed black line) and the exponential part (dashed-dotted blue line) of $U_{i}(t)$ are plotted separately. The dotted red line which is the extrapolation of the fit of the $1^{\text {st }}$ sequence demonstrates that the voltage decays within all other sequences (except $i=5$ ) approach this line asymptotically with time.

The fit parameters determined for each FC-DLC sequence are plotted versus the sequence index $i$ in Figure 5 (B) to evaluate the contribution of the individual parameters (to the reversible degradation) over time. At BoT the exponential contribution is much smaller than at EoT. Specifically, an almost linear increase of the amplitude $c_{i}$ from $0.04 \mathrm{~V}$ to about $0.14 \mathrm{~V}$ is observed in the course of the test. The decay constant $\lambda_{i}$ increases too, from $0.015 \mathrm{~h}^{-1}$ in the first sequence to almost $0.07 \mathrm{~h}^{-1}$ in sequence 5 . The increase is strongest from sequence $i=1$ to $i=2$ and linear afterwards. Specifically, the exponential decay at EoT is approximately 4.5 times faster (increase of $\lambda_{i}$ ) and 3.5 more intensive (increase of $c_{i}$ ) than at BoT. In the fourth and fifth sequence, the increase of $\lambda_{i}$ and $c_{i}$ is not amplified, suggesting that the exponential part of $U_{i}(t)$ is not related to the MEA failure (see Figure 2).

In contrast to the exponential parameters, a sudden increase of the slope $b_{i}$ of the linear degradation in the $5^{\text {th }}$ sequence can be positively related to MEA failure. Before $b_{i}$ is influenced by this failure, its value increases from $0.28 \mathrm{mV} \mathrm{h}^{-1}$ in sequence $i=1$ to $0.45 \mathrm{mV} \mathrm{h}^{-1}$ in sequence $i=2$ and is afterwards almost constant between 0.45 and $0.50 \mathrm{mV} \mathrm{h}^{-1}$. It is noted that the linear decay rate $\left(b_{i}\right)$ includes contributions of reversible $\left(b_{i, \text { rev }}\right)$ and irreversible $\left(b_{i, \text { irrev }}\right)$ degradation, i.e. $b_{i}=b_{i, \text { rev }}+b_{i, \text { irrev }}$. Thereby, $b_{i, \text { irrev }}$ can be determined from a linear regression of the voltage values after each refresh as described in section 3.2. For MEA1 at $1 \mathrm{~A} \mathrm{~cm}^{-2}, b_{i, \text { irrev }}$ was determined from Figure 2 to $0.05 \mathrm{mV} \mathrm{h}^{-1}$, being a constant offset of $b_{i}$. 


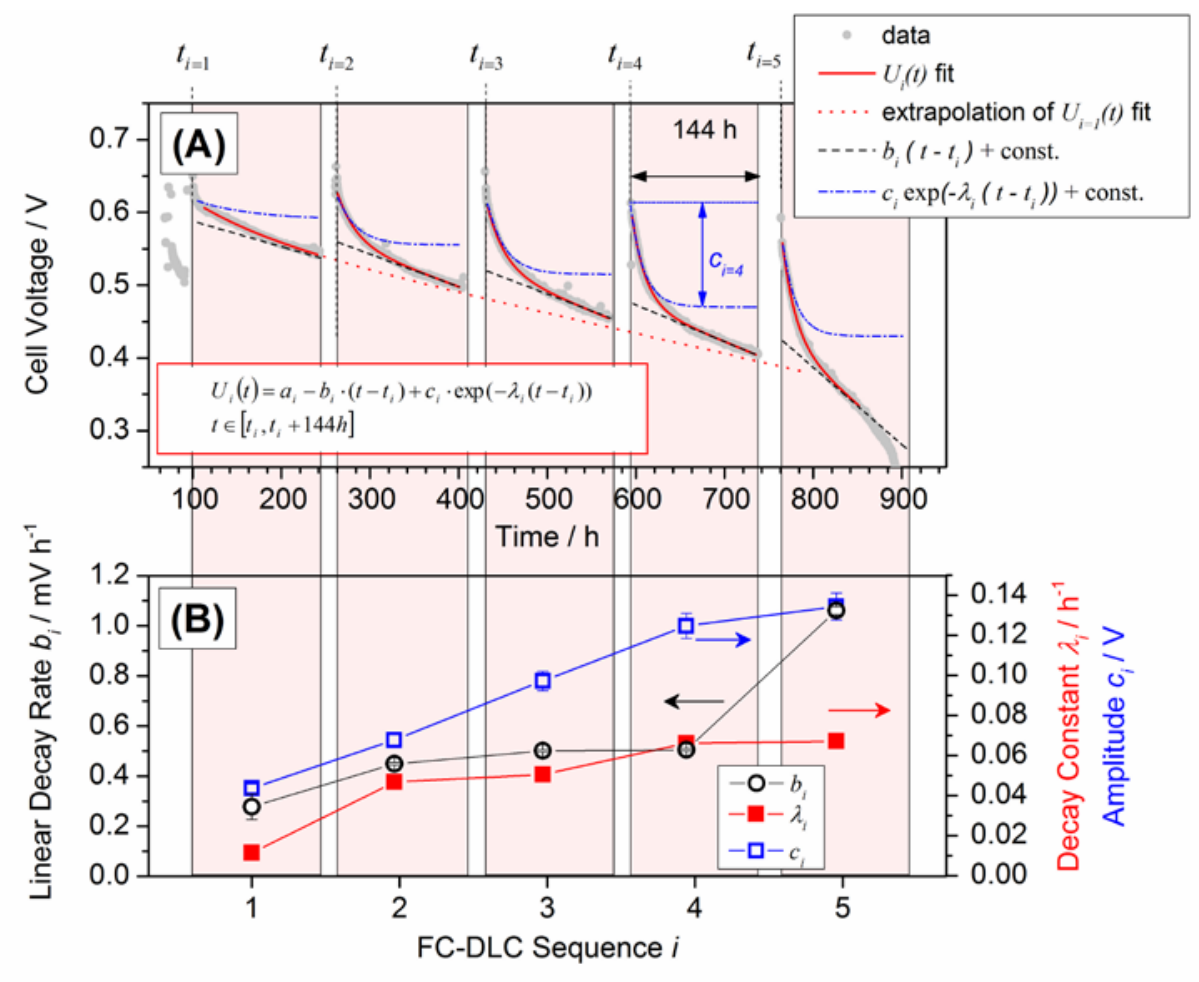

Figure 5: (A) Voltage values of MEA1 corresponding to $1 \mathrm{~A} \mathrm{~cm}^{-2}$ load (grey data), linear-exponential fit of the data (solid red curves) as well as the linear (dashed black lines) and the exponential (dashed-dotted blue curve) part of the fit (the linear and the exponential parts are offset vertically for a better clarity of presentation). The dotted red line is the extrapolation of $U_{i=1}$. (B) linear decay rate $b_{i}$, decay constant $\lambda_{i}$, and the amplitude $c_{i}$ of the exponential term determined from the linear-exponential fits for each FC-DLC sequence.

A comparison of the parameters $b_{i}, \lambda_{i}$, and $c_{i}$ of the function $U_{i}(t)$ fitted to the voltage of different load levels (current densities) within individual sequences $i$ are provided in Figure 6 . Generally, the linear voltage decay rates $b_{i}$ in panel (A) increase from FC-DLC sequence $i=1$ to sequence $i=2$ and are almost constant afterwards. For $i=1 \ldots 4$ all $b_{i}$ values for the different loads are in the range 0.3 to $0.6 \mathrm{mV} \mathrm{h}^{-1}$. Hence, the significant increase of the reversible degradation rate over time is not a result of a change of the linear contribution. Rather, it must be a consequence of the exponential contribution instead. In sequence $i=5$ the values corresponding to $j=0.8$ and $1 \mathrm{~A} \mathrm{~cm}^{-2}$ behave differently compared to the others, i.e. they exhibit a sudden strong increase related to the failure of the MEA. In contrast to the other load levels, the $b_{i}$ values for $0.05 \mathrm{~A} \mathrm{~cm}^{-2}$ increase almost linearly over time. The behavior of the values for the other load levels is comparable.

In the case of the decay constants $\lambda_{i}$ plotted in panel (B), the values for $i=1$ are not conclusive due to their large uncertainty caused by the vanishing amplitude $c_{i}$ of the exponential part in the first test sequence (compare panel (C)). Nevertheless, for $i=2-5$ the exponential decay constants behaves similarly for all loads being in the range $0.4-0.7 \mathrm{~h}^{-1}$. No clear trend for an increase with operation time is observed. The decay constants $\lambda_{i}$ represents a reciprocal time constant and specifies the duration of the exponential degradation period in each FC-DLC sequence; the lower its value, the longer this period. Consequently, the duration of the impact of the exponential contribution is not clearly prolonged or shortened in the presented test.

For the amplitude of the exponential part depicted in panel (C), on the other hand, a clear increase is observed with operation time; for all loads it starts at low values $c_{i=1}<0.05 \mathrm{~V}$ and increases linearly with $i$ up to $c_{i=5}=0.12-0.15 \mathrm{~V}$. This linear increase demonstrates that for a cell exhibiting non- 
constant reversible degradation (MEA1) the exponential contribution to $U_{i}(t)$ becomes significantly larger over time and the degradation behavior of the examined cell becomes more and more dominated by the exponential function.

In summary, the exponential term of the proposed fit function strongly influences the degradation behavior, especially during the first operating hours after shutdown and with increasing lifetime of the fuel cell. Up to now, the use of one single value based on a linear regression is common to describe degradation rates. The given example, however, demonstrates the importance of the exponential contribution to predict a certain cell performance after a few operating hours - a scenario of special interest for the automotive application of fuel cells.
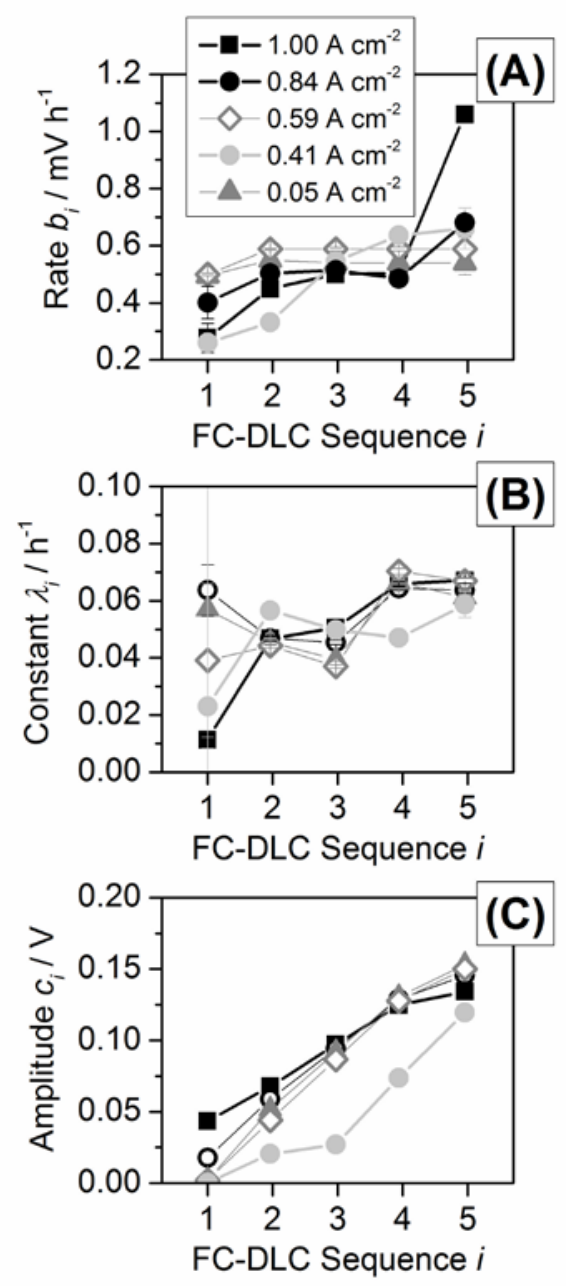

Figure 6: Fit parameters of the linear-exponential fit of the voltage decay within the individual sequences $i$ analyzed for different current densities. (A) slope $b_{i}$ of the linear decay, (B) decay constant $\lambda_{i}$ of the exponential part of the decay, (C) amplitude $c_{i}$ of the exponential part of the decay.

\subsection{Recovery of Reversible Voltage Losses}

As seen in section 3.2, the reversible degradation has a significant impact on the differential resistance at low current densities. It is known that lower performance at low electrical load can be caused by increased gas crossover resulting in the formation of mixed potentials and/or decreased catalyst activity $[27,28]$. Consequently, there could be several reasons for the observed reversible voltage decay and different strategies for recovery of the cell performance: 
(i) Water can be accumulated in the electrodes partially blocking the mass-transport of the reactants to the catalysts. The increased concentration over-potential can significantly decrease the cell performance [29]. A recovery procedure requires the removal of the water by electrodes flushing with dry gases.

(ii) The membrane can be damaged during operation resulting in the formation of pinholes and micro cracks. The increased gas crossover may cause mixed potentials at the electrodes, especially at low current densities. As shown by Kreitmeier et al. [24], liquid product water can seal membrane defects and eliminate the crossover during fuel cell operation. On the contrary, drying condition can result in membrane shrinking, which may enlarge micro cracks and increase the gas crossover [30]. Consequently, a recovery procedure for membrane effects could include an increase of the cell humidity and/or a decrease of the mechanical stress applied to the membrane.

(iii) The surface of the anodic catalyst can be covered by contaminant, e.g. by CO present in the fuel. The resulting decrease of active catalyst sites can decrease the hydrogen reduction kinetic and increase mass transport effects at the anode. If pure platinum catalysts are used, an anode potential higher than $0.6 \mathrm{~V}$ is required for the oxidative CO removal, a potential not present at the anode during fuel cell operation [31]. A possibility to recover the performance loss caused by anode poisoning is the increase of the anodic potential during cell shutdown.

(iv) The formation of platinum oxides on the surface of the catalyst can decrease the electro-catalytic activity of the oxygen reduction reaction at the cathode. This oxide formation is known to be present at high cathode potential during low current densities and OCV. A cathode potential below $0.65 \mathrm{~V}$ is required for the reduction of the platinum oxide. This potential is present during fuel cell operation at high load only [32]. Moreover, contaminants from ionomer degradation may block the cathodic catalyst and lower the cell performance [8]. The resulting recovery strategy for both effects is the decrease of the cathodic potential during shutdown.

The recovery of the reversible performance losses was analyzed based on a durability test performed using MEA2 (see supporting information Figure S1). The test is similar to the one described in subsection 3.1 for MEA1. The test protocol was the FC-DLC and the cell operation was interrupted after $100-200 \mathrm{~h}$ of operation by a shutdown recovery procedure, assumed to recover the entire reversible degradation effects. The test bench parameters recorded during a shutdown recovery procedure are provided in Figure 7. This procedure consists of switching off the load, stopping the gas supply, opening gas outlet valves (bringing cell to ambient pressure and allowing air to enter the electrode compartments by diffusion), and letting the cell cool down to room temperature. At the beginning of this procedure the cell voltage remains for several minutes to tens of minutes at OCV due to the remaining hydrogen and air at anode and cathode, respectively. Eventually, the cell voltage drops to values close to $0 \mathrm{~V}$ with slight fluctuations up to approx. $150 \mathrm{mV}$, representing air at both electrodes (high electrode potential at anode and cathode). After waiting several hours (typically one night) the cell is switched on again and the FC-DLC test is being continued. 


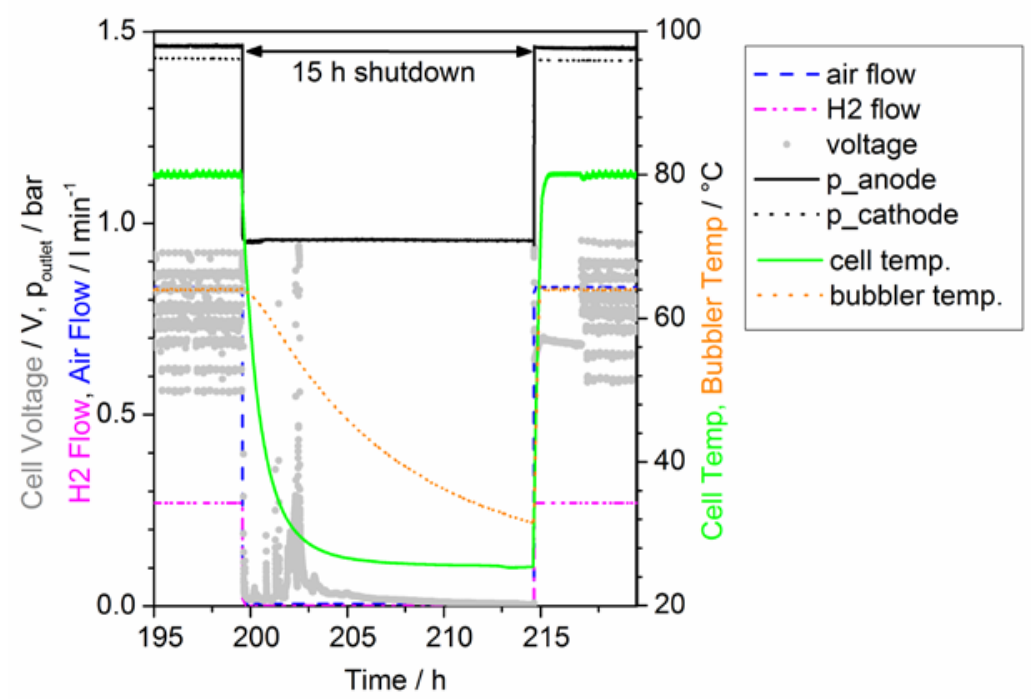

Figure 7: Test bench parameters recorded during a shutdown procedure for recovery of reversible losses.

To study the effect of different shutdown parameters on recovery of reversible losses, the cell operation was interrupted by several procedures of approx. $1 \mathrm{~h}$ partially imitating conditions that occur during a shutdown. These recovery test procedures are listed in Table 1 along with a qualitative assessment of their impact on recovery. To quantitatively determine the voltage recovery at the different current densities for a certain test procedure the voltage values before the refresh and after $1 \mathrm{~h}$ operation after the refresh (to let the cell equilibrate) are compared. The difference of these voltages is defined as recovered voltage. The voltages recovered by the different procedures versus the current density are plotted in Figure $8(A)-(D)$. The recovery by the shutdown recovery procedure (assumed as full recovery and visualized by black bullets) shows a minimum recovery of around $20 \mathrm{mV}$ at low current densities and a maximum of $40 \mathrm{mV}$ at $0.8 \mathrm{~A} \mathrm{~cm}^{-2}$. It is included in all panels to allow a comparison with the other procedures.

Table 1: Voltage recovery test procedures analyzed during the durability test of MEA2.

\begin{tabular}{|c|c|c|c|}
\hline Recovery test & Intention & $\begin{array}{c}\text { Recovered } \\
\text { voltage @ 0.2 } \\
\mathbf{A ~ \text { cm} ^ { - 2 }}\end{array}$ & $\begin{array}{c}\text { Recovered } \\
\text { voltage @ 0.8 } \\
\mathbf{A ~ c m}^{-2}\end{array}$ \\
\hline $\begin{array}{c}\text { Purging anode } \\
\text { with dry } \mathrm{H}_{2}\end{array}$ & Remove water from anode & $-28 \%$ & $-20 \%$ \\
\hline $\begin{array}{c}\text { Purging cathode } \\
\text { with dry air }\end{array}$ & Remove water from cathode & $19 \%$ & $-10 \%$ \\
\hline $\begin{array}{c}\text { Reduction of cell } \\
\text { temperature }\end{array}$ & $\begin{array}{c}\text { Increase humidity and decrease } \\
\text { mechanical membrane stress }\end{array}$ & $38 \%$ & $68 \%$ \\
\hline OCV-Test & $\begin{array}{c}\text { Drying of MEA and increase of } \\
\text { cathode potential }\end{array}$ & $0 \%$ & $20 \%$ \\
\hline $\begin{array}{c}\text { Purging anode } \\
\text { with air }\end{array}$ & $\begin{array}{c}\text { Increase anode potential to } \\
\text { remove contaminants }\end{array}$ & $19 \%$ & $10 \%$ \\
\hline $\begin{array}{c}\text { Stopping gas flow } \\
\text { Increase anode potential to } \\
\text { remove contaminants }\end{array}$ & $0 \%$ & $21 \%$ \\
\hline $\begin{array}{c}\text { Purging cathode } \\
\text { with } \mathrm{N}_{2}\end{array}$ & $\begin{array}{c}\text { Decrease cathode potential to } \\
\text { reduce platinum oxide and remove } \\
\text { contaminants }\end{array}$ & $14 \%$ & \\
\hline
\end{tabular}


For the assessment of water accumulation in both electrodes (i), the cell was purged with dry hydrogen and dry air in two separate shutdown experiments, while the other parameters were kept constant. No recovery effect could be observed and the resulting voltage after cell restart was even lower due to decreased humidity level of the ionomer as observed in see Figure 8 (A). Consequently, water accumulation seems not to contribute to the voltage recovery effect.

The impact of ionomer effects on voltage recovery was examined by reducing the cell temperature while keeping the cell at OCV to increase the relative humidity inside the cell and to reduce the mechanical stress to the membrane, see Figure 8 (B). Thereby, both the ionomer in the catalyst layer and the membrane may be affected. As additionally shown, the recovery effect was not influenced by the OCV itself. The temporary reduction of the cell temperature has a significant effect on recovery. However, voltage losses at very low loads are not recovered. At current densities higher 0.2 $\mathrm{A} \mathrm{cm}^{-2}$ around $50-70 \%$ of the full recovery is reached. The effect is not simply a rehydration of the ionomer, but it seems to be a combination of several interacting effects which are not distinguish in this paper.

For the evaluation of the anode catalyst effect (iii) two tests indicated in Figure 8 (C) were realized to increase the anodic potential. The anode was actively purged with humidified air and the reactant flow was stopped to allow air entering the anode by diffusion, respectively. The voltage recovery of 5 $-10 \mathrm{mV}$ (approx. 10 - $20 \%$ of full recovery) by both tests was comparable with slightly higher effects for the active purge. This affects the entire current density range from OCV to $1.0 \mathrm{~A} \mathrm{~cm}^{-2}$ with an almost linear increase over current density. The minor effect by stopping the gas flows can be explained by a decreased recovery time at high anode potential limited by diffusion of the air into the anode compartment.

The effect of low cathode potentials on the recovery effect was analyzed by flushing the cathode with nitrogen while the anode supply with hydrogen was maintained. During this experiment a cathode potential of approximately $70 \mathrm{mV}$ was achieved. A minor voltage recovery of $\sim 5 \mathrm{mV}$ (approx. $15-20 \%$ of full recovery) could be detected, see Figure 8 (D).

In summary, the high recovery of reversible losses obtained by a shutdown recovery procedure seems to be the result of different effects. Thereby, the above data indicates that ionomer effects have the highest impact on the performance recovery of reversible voltage losses. Additionally, the presence of contaminants at the anode demonstrated a minor but significant impact too. The sum of the effects due to purging the anode with air and temporarily reducing cell temperature is shown in panel (E). This combination of high anode potential for contaminant removal and reduced mechanical stress to the membrane leads, at least for high current densities, to the full recovery effect achieved by the shutdown recovery procedure. Since the cathode potential is not lowered during the shutdown recovery, the minor impact of the decreased cathode potential is not included in this plot. For low loads, however, the recovery achieved by the shutdown recovery procedure could not be reproduced by any other procedure. 

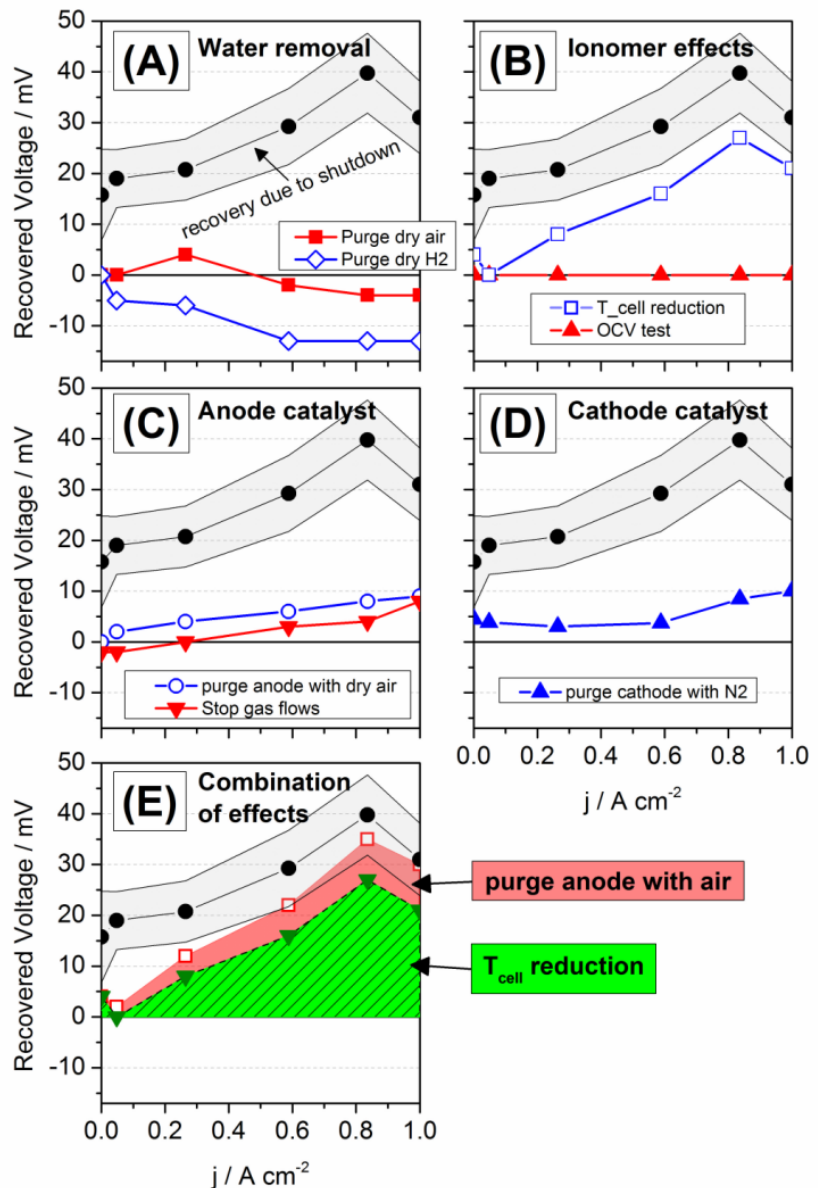

$\mathrm{j} / \mathrm{A} \mathrm{cm}^{-2}$

purge anode with air

cell reduction

Figure 8: (A) - (D) Recovered voltage versus current density due to different procedures indicated in the individual panels. In the case of recovery by shutdown (bullets) the grey area corresponds to the standard deviation from the mean of four different shutdown events. (E) Comparison of the voltage recovery due to shutdown and the added up recoveries due to reduction of cell temperature and purging the anode with air.

\section{CONCLUSIONS}

The FC-DLC test interrupted by shutdown recovery procedures is a useful approach to discriminate between reversible and irreversible degradation and, simultaneously, obtain data for different load levels; i.e. the test allows a quasi-simultaneous acquisition of voltage values at the different current densities defined in the test protocol allowing for the determination of the current density dependent degradation rates. It is proposed to report MEA degradation rates as voltage loss diagrams showing degradation rates versus current density.

Irreversible degradation rates cannot be determined from short test (e.g. 100 h). Rather, extended durability test with several refresh interruptions are needed. In agreement with this observation the irreversible degradation rates can be most reliably determined by linearly fitting the voltage values directly after refresh steps. The degradation rates evaluated using the voltage values directly before performing a refresh, on the other hand, are not always identical with the irreversible degradation; for non-constant reversible degradation, this methods yields a mixed reversible-irreversible degradation rate not allowing to isolate the irreversible one.

The reversible degradation occurring upon continuous FC-DLC operation in between the shutdown recovery procedures has been quantitatively described by a linear-exponential function. The 
degradation rate during the first operation hours of a fuel cell after restart is dominated by the exponential term, especially by the amplitude of the exponential term, and the commonly used calculation by linear regression does not adequately describe the degradation effect during these highly important hours for automotive application. For the tested MEA the linear part of the reversible decay could be correlated with a sudden physical damage of the membrane.

According to the best of our knowledge for the most commonly used type of MEAs (Pt/C and PFSA based), the shutdown recovery procedure, basically being a total shutdown of the cell with stopping of gas supply and reduction of cell temperature to room temperature, leads to the full recovery of reversible voltage losses. The membrane defects (possibly pinholes) responsible for low OCV values can also be temporarily reduced by the shutdown recovery likely due to sealing of the pinholes by water. It is last to mention that for other kind of materials, the behavior may be different and the shutdown recovery procedure may not lead to fully recovery of reversible losses.

\section{Acknowledgements}

The research leading to these results has received funding from the European Union's Seventh Framework Programme (FP7/2007-2013) for Fuel Cell and Hydrogen Joint Technology Initiative under Grant $n^{\circ} 303452$ (Impact). The authors thank Siegfried Graf for setting up the fuel cell test stations.

\section{References}

[1] R. Borup, J.R. Davey, F.H. Garzon, D.L. Wood, M.A. Inbody, PEM fuel cell electrocatalyst durability measurements, J. Power Sources. 163 (2006) 76.

[2] R. Borup, J. Meyers, B. Pivovar, Y.S. Kim, R. Mukundan, N. Garland, et al., Scientific aspects of polymer electrolyte fuel cell durability and degradation., Chem. Rev. 107 (2007) 3904.

[3] R. Borup, R. Mukundan, PEM Fuel Cell Degradation, ECS Trans. 33 (2010) 17.

[4] M. Schulze, K.A. Friedrich, Relations of Water Management and Degradation Processes in PEFC, ECS Trans. 12 (2008) 101.

[5] F.A. De Bruijn, V.A.T. Dam, G.J.M. Janssen, Review: Durability and degradation issues of PEM fuel cell components, Fuel Cells. 8 (2008) 3-22.

[6] W. Schmittinger, A. Vahidi, A review of the main parameters influencing long-term performance and durability of PEM fuel cells, J. Power Sources. 180 (2008) 1.

[7] N. Ramaswamy, N. Hakim, S. Mukerjee, Degradation mechanism study of perfluorinated proton exchange membrane under fuel cell operating conditions, Electrochim. Acta. 53 (2008) 3279-3295.

[8] A. Kongkanand, J. Zhang. Z. Liu, Y.-H. Lai, P. Sinha, E.L. Thompson, R. Makharia, Degradation of PEMFC Observed on NSTF Electrodes, J. Electrochem. Soc. 161 (2014) F744-F753.

[9] S. Helmly, B. Ohnmacht, P. Gazdzicki, R. Hiesgen, E. Gulzow, K.A. Friedrich, Influence of the Distribution of Platinum Deposits on the Properties and Degradation of Platinum-Impregnated Nafion Membranes, J. Electrochem. Soc. 161 (2014) F1416-F1426.

[10] S. Kundu, M. Fowler, L.C. Simon, R. Abouatallah, Reversible and irreversible degradation in fuel cells during Open Circuit Voltage durability testing, J. Power Sources. 182 (2008) 254-258.

[11] A. Panchenko, H. Dilger, E. Möller, T. Sixt, E. Roduner, In situ EPR investigation of polymer electrolyte membrane degradation in fuel cell applications, J. Power Sources. 127 (2004) 325330. 
[12] R. Petrone, D. Hissel, M.C. Péra, D. Chamagne, R. Gouriveau, Accelerated stress test procedures for PEM fuel cells under actual load constraints: State-of-art and proposals, Int. J. Hydrogen Energy. 40 (2015) 12489-12505.

[13] M. Kim, N. Jung, K. Eom, S.J. Yoo, J.Y. Kim, J.H. Jang, et al., Effects of anode flooding on the performance degradation of polymer electrolyte membrane fuel cells, J. Power Sources. 266 (2014) 332-340.

[14] S. Enz, T.A. Dao, M. Messerschmidt, J. Scholta, Investigation of degradation effects in polymer electrolyte fuel cells under automotive-related operating conditions, J. Power Sources. 274 (2015) 521-535.

[15] M. Schulze, N. Wagner, T. Kaz, K.A. Friedrich, Combined electrochemical and surface analysis investigation of degradation processes in polymer electrolyte membrane fuel cells, Electrochim. Acta. 52 (2007) 2328.

[16] J. Zhang, B.A. Litteer, F.D. Coms, R. Makharia, Recoverable Performance Loss Due to Membrane Chemical Degradation in PEM Fuel Cells, J. Electrochem. Soc. 159 (2012) F287F293.

[17] A. El-Kharouf, A. Chandan, M. Hattenberger, B.G. Pollet, Proton exchange membrane fuel cell degradation and testing: review, J. Energy Inst. 85 (2012) 188-200.

[18] H. Cha, C. Chen, J. Shiu, Investigation on the durability of direct methanol fuel cells, J. Power Sources. 192 (2009) 451-456.

[19] F. Bresciani, C. Rabissi, M. Zago, P. Gazdzicki, M. Schulze, L. Guétaz, et al., A combined in-situ and post-mortem investigation on local permanent degradation in a direct methanol fuel cell, J. Power Sources. 306 (2016) 49-61.

[20] G. Tsotridis, A. Pilenga, G. De Marco, T. Malkow, EU Harmonised Test Protocols for PEMFC MEA Testing in Single Cell Configuration for Automotive Applications; JRC Science for Policy report, (2015) EUR $27632 \mathrm{EN}$; doi 10.2790/54653.

[21] Stack-Test Test Module D-02 “Load Cycling Durability" http://stacktest.zsw-bw.de/mediacentre.html, 2016.

[22] H. Lee, T. Kim, W. Sim, S. Kim, B. Ahn, T. Lim, et al., Pinhole formation in PEMFC membrane after electrochemical degradation and wet/dry cycling test, Korean J. Chem. Eng. 28 (2011) 487-491.

[23] T. V. Reshetenko, G. Bender, K. Bethune, R. Rocheleau, Application of a segmented cell setup to detect pinhole and catalyst loading defects in proton exchange membrane fuel cells, Electrochim. Acta. 76 (2012) 16-25.

[24] S. Kreitmeier, M. Michiardi, A. Wokaun, F.N. Büchi, Factors determining the gas crossover through pinholes in polymer electrolyte fuel cell membranes, Electrochim. Acta. 80 (2012) 240-247.

[25] S. Kreitmeier, G.A. Schuler, A. Wokaun, F.N. Büchi, Investigation of membrane degradation in polymer electrolyte fuel cells using local gas permeation analysis, J. Power Sources. 212 (2012) 139-147.

[26] T.J. Mason, J. Millichamp, P.R. Shearing, D.J.L. Brett, A study of the effect of compression on the performance of polymer electrolyte fuel cells using electrochemical impedance spectroscopy and dimensional change analysis, Int. J. Hydrog. Energy. 38 (2013) 7414-7422.

[27] S.S. Kocha, J.D. Yang, Jung S. Yi, Characterization of Gas Crossover and Its Implications in PEM Fuel Cells, AIChE J. 52 (2006) 1916-1925. 
[28] A.Z. Weber, Gas-Crossover and Membrane-Pinhole Effects in Polymer-Electrolyte Fuel Cells, J. Electrochem. Soc. 155 (2008) B521.

[29] N. Yousfi-Steiner, P. Moçotéguy, D. Candusso, D. Hissel, A. Hernandez, A. Aslanides, A review on PEM voltage degradation associated with water management: Impacts, influent factors and characterization, J. Power Sources. 183 (2008) 260-274.

[30] Y.-H. Lai, C.K. Mittelsteadt, C.S. Gittleman, D.A. Dillard, Viscoelastic Stress Analysis of Constrained Proton Exchange Membranes Under Humidity Cycling, J. Fuel Cell Sci. Techn. 6 (2009) 021002.

[31] X. Cheng, Z. Shi, N. Glass, L. Zhang, J. Zhang, D. Song, et al., A review of PEM hydrogen fuel cell contamination: Impacts, mechanisms, and mitigation, J. Power Sources. 165 (2007) 739756.

[32] S.G. Rinaldo, W. Lee, J. Stumper, M. Eikerling, Mechanistic Principles of Platinum Oxide Formation and Reduction, Electrocatalysis. 5 (2014) 262-272. 


\title{
SUPPORTING INFORMATION
}

\section{FOR}

\section{Evaluation of reversible and irreversible degradation rates of polymer electrolyte membrane fuel cells tested in automotive conditions}

\author{
Pawel Gazdzick $^{1}$, Jens Mitzel ${ }^{1}$, Daniel Garcia Sanchez ${ }^{1}$, Mathias Schulze ${ }^{1}$, K. Andreas Friedrich ${ }^{1,2}$ \\ ${ }^{1}$ German Aerospace Center, Institute of Engineering Thermodynamics, Pfaffenwaldring 38-40, 70569 Stuttgart, \\ Germany \\ ${ }^{2}$ University of Stuttgart, Institute of Energy Storage, Pfaffenwaldring 31, 70569 Stuttgart, Germany \\ *Corresponding author \\ email address: pawel.gazdzicki@dlr.de (P. Gazdzicki)
}

Figure S1 shows the durability test of MEA 2 performed using the FC-DLC protocol. To study the effect of the shutdown on recovery of reversible losses the cell operation was interrupted by different procedures (indicated by colored boxes) imitating conditions that occur during a shutdown that include holding the cell at OCV, stopping gas supply, purging the cell with $\mathrm{N}_{2}$, changing cell potential, purging with dry reactant gases, temporarily reducing the cell temperature.

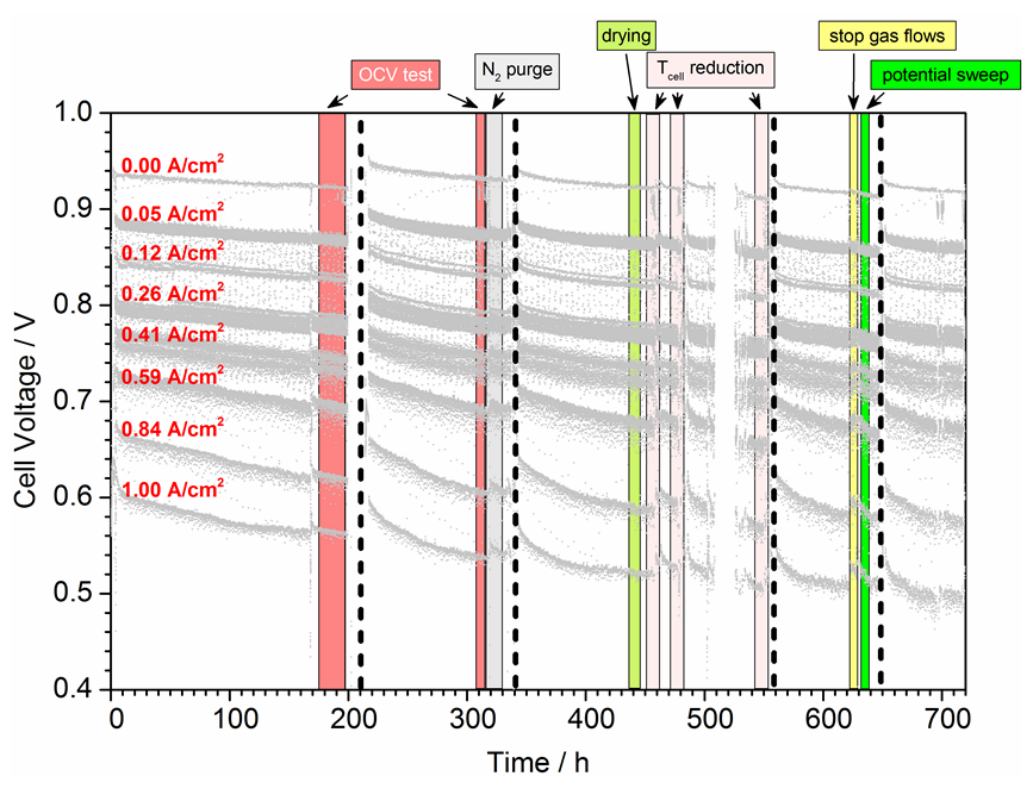

Figure S1: FC-DLC durability test of MEA2 interrupted by shutdown procedure (vertical dashed lines) and by different recovery tests indicated in the figure by the colored boxes. The test was performed at $80^{\circ} \mathrm{C}$ cell temperature, constant flow rates of $260 \mathrm{ml} \mathrm{min}^{-1}\left(\mathrm{H}_{2}\right)$ and $830 \mathrm{ml} \mathrm{min}^{-1}$ (air). The humidity was $50 \% \mathrm{RH}$ during the first $700 \mathrm{~h}$ and $80 \% \mathrm{RH}$ afterwards as indicated in the figure. At the right left side of the plot current density values belonging to the different voltage levels are indicated. 